\title{
Measuring Entropy Change in a Human Physiological System
}

\author{
Satish Boregowda, ${ }^{1}$ Rod Handy, ${ }^{2}$ Darrah Sleeth, ${ }^{2}$ and Andrew Merryweather ${ }^{3}$ \\ ${ }^{1}$ School of Mechanical Engineering, Purdue University, West Lafayette, IN 47907, USA \\ ${ }^{2}$ Department of Family \& Preventive Medicine, University of Utah, Salt Lake City, UT 84108, USA \\ ${ }^{3}$ Department of Mechanical Engineering, University of Utah, Salt Lake City, UT 84108, USA \\ Correspondence should be addressed to Rod Handy; rod.handy@hsc.utah.edu
}

Received 11 November 2015; Revised 2 January 2016; Accepted 4 January 2016

Academic Editor: Felix Sharipov

Copyright (c) 2016 Satish Boregowda et al. This is an open access article distributed under the Creative Commons Attribution License, which permits unrestricted use, distribution, and reproduction in any medium, provided the original work is properly cited.

\begin{abstract}
The paper presents a novel approach involving the use of Maxwell relations to combine multiple physiological measures to provide a measure of entropy change. The physiological measures included blood pressure (BP), heart rate (HR), skin temperature (ST), electromyogram (EMG), and electrodermal response (EDR). The multiple time-series physiological data were collected from eight subjects in an experimental pilot study conducted at the Human Engineering Laboratory of NASA Langley Research Center. The methodology included data collection during a relaxation period of eighteen minutes followed by a sixty-minute cognitive task. Two types of entropy change were computed: (a) entropy change $\left(\Delta S_{\mathrm{BP}}\right)$ due to blood pressure, heart rate, and skin temperature and (b) entropy change $\left(\Delta S_{\mathrm{EMG}}\right)$ due to electromyogram, electrodermal response, and skin temperature. The results demonstrate that entropy change provides a valuable composite measure of individual physiological response to various stressors that could be valuable in the areas of medical research, diagnosis, and clinical practice.
\end{abstract}

\section{Introduction}

Earlier work done by several researchers has established the fact that human life processes are indeed thermodynamic in nature and hence thermodynamic laws can be used to model human physiology. It has been hypothesized by Bridgman [1] that the laws of thermodynamics are intrinsic to nature and life and are thus well positioned to characterize the physiological behavior of living systems. Aoki [2,3] examined human thermoregulation by measuring entropy flow and production in basal and exercising conditions but did not utilize Maxwell relations to include other physiological responses. The seminal work done in the areas of biological thermodynamics by Morowitz $[4,5]$ does not show any application of Maxwell relations to model human physiology. Furthermore, the work done by Garby and Larsen [6] and Jou and Llebot [7] addresses mass, energy, and entropy balance of open living systems but not Maxwell relations. However, the detailed development and preliminary verification of physiological entropy change using Maxwell relations have been presented by Boregowda et al. $[8,9]$ and are reproduced in Appendix for the convenience of the readers. The present study utilizes time-series data collected during a cognitive task to validate and verify entropy change metrics representing two human physiological subsystems. For the convenience of the readers, the detailed development of physiological entropy change using Maxwell relations is presented in Appendix of this paper. The first one combines changes in blood pressure, heart rate, and skin temperature to provide a BP-based entropy change $\left(\Delta S_{\mathrm{BP}}\right)$. The second one combines changes in electromyogram, electrodermal response, and skin temperature to provide an EMG-based entropy change $\left(\Delta S_{\mathrm{EMG}}\right)$.

\section{Modeling and Formulation}

2.1. Entropy Change $\left(\Delta S_{\mathrm{BP}}\right.$ : Blood Pressure, Heart Rate, and Skin Temperature). Let us first consider the human physiological subsystem characterized by blood pressure (BP), heart rate (HR), and skin temperature (ST) as a closed thermodynamic system. For this system, the work done by the system is given by [10]

$$
\delta W=P * d V=\mathrm{BP} * d(\mathrm{HR}),
$$


where $P$ is pressure, BP is blood pressure $(\mathrm{mmHg}), V$ is volume, and $\mathrm{HR}$ is heart rate (beats/minute).

The heart rate is an indirect measure of stroke volume of blood in the heart region and is, thus, used in the place of stroke volume. Please note that heart rate (in beats per minute) is equal to cardiac output $(\mathrm{mL} / \mathrm{min})$ divided by stroke volume $(\mathrm{mL} / \mathrm{beat})$. The ratio of partial changes in two physiological variables is accompanied by constancy in the third variable, as this is the basis of Maxwell relations. For example, when there is a partial change in heart rate with respect to partial change in entropy, the mean arterial pressure remains constant. As shown in Appendix, the thermodynamic potential is derived as shown starting with Helmholtz's potential $(F=U-T S)$ and arriving with the following property relation:

$$
d F=-\mathrm{BP} * d(\mathrm{HR})-S * d(\mathrm{ST}) .
$$

The following Maxwell relation is obtained by invoking the exactness condition to the above property relation (2):

$$
\left(\frac{\partial \mathrm{BP}}{\partial \mathrm{ST}}\right)_{V}=\left(\frac{\partial S}{\partial \mathrm{HR}}\right)_{T} \text {. }
$$

The partial derivative is approximated to form a modified Maxwell relation that is used in the present experimental study to compute the physiological entropy change resulting from changes in blood pressure (BP), heart rate (HR), and skin temperature (ST):

$$
\begin{aligned}
\left(\frac{\Delta \mathrm{BP}}{\mathrm{ST}}\right)_{\mathrm{HR}} & =\left(\frac{\Delta S_{\mathrm{BP}}}{\Delta \mathrm{HR}}\right)_{\mathrm{ST}}, \\
\Delta S_{\mathrm{BP}} & =\frac{\Delta \mathrm{BP} \times \Delta \mathrm{HR}}{\Delta \mathrm{ST}} .
\end{aligned}
$$

The skin temperature (ST) in the denominator acts as a temperature at the boundary of the closed thermodynamic system and provides a physiological reflection of emotional response. If one were to consider either internal energy or the Gibbs function to define human physiological function, then one would get a negative sign preceding the entropy change $\left(\Delta S_{\mathrm{BP}}\right)$. The deviation from the relaxed state may be positive or negative depending on the imposed external stressor and the internal physiological condition.

2.2. Entropy Change ( $\Delta S_{\mathrm{EMG}}$ : Electromyogram, Electrodermal Response, and Skin Temperature). Let us consider the human physiological subsystem characterized by electromyogram (EMG), electrodermal response (EDR), and skin temperature (ST) as a closed thermodynamic system. For this system, the work done by the system is given by

$$
\delta W=\llbracket * d L=\mathrm{EMG} * d(\mathrm{EDR}),
$$

where $\llbracket$ is tension, EMG is facial electromyogram $(\mathrm{mV}), L$ is length, and EDR is electrodermal response $(\mathrm{mV})$.

In this regard, the elements of a human physiological subsystem system are considered as equivalent to a conducting wire with $\mathbb{a}$ as tension and $L$ as the length. The facial electromyogram (EMG) has been used in medical research and clinical practice as a measure of tension in facial muscles [11]. As a result of tension in muscles or nerves, the outer surface of the skin responds by expanding or contracting in terms of changes called electrodermal response (EDR). The skin temperature (ST) represents the thermal response to tension. The thermodynamic potential is again derived, starting with Helmholtz's potential $(F=U-T S)$ arriving at the following property relation:

$$
d F=-\mathrm{EMG} * d(\mathrm{EDR})-S * d(\mathrm{ST}) .
$$

The following Maxwell relation is obtained by invoking the exactness condition to the above property relation (2):

$$
\left(\frac{\partial \mathrm{EMG}}{\partial \mathrm{ST}}\right)_{\mathrm{EDR}}=\left(\frac{\partial S}{\partial \mathrm{EDR}}\right)_{\mathrm{ST}} .
$$

The partial derivative is approximated to form a modified Maxwell relation that is used in the present experimental study to compute the physiological entropy change resulting from changes in blood pressure (BP), heart rate (HR), and skin temperature (ST):

$$
\begin{aligned}
\left(\frac{\Delta \mathrm{EMG}}{\mathrm{ST}}\right)_{\mathrm{EDR}} & =\left(\frac{\Delta S_{\mathrm{EMG}}}{\Delta \mathrm{EDR}}\right)_{\mathrm{ST}}, \\
\Delta S_{\mathrm{EMG}} & =\frac{\Delta \mathrm{EMG} \times \Delta \mathrm{EDR}}{\Delta \mathrm{ST}} .
\end{aligned}
$$

As mentioned earlier, the use of either internal energy or the Gibbs function would have resulted in a negative sign preceding the entropy change $\left(\Delta S_{P}\right)$. The deviation from the relaxed state may be positive or negative, depending on the imposed external stressor and the internal physiological condition. Specifically, physiological entropy is qualitatively defined as a measure of disorder [12]. It is a kind of global measure that specifies how violent motions and reactions are occurring in nature. Hence, the entropy change in the human physiological system shows the extent of activity within the body as a whole; thus, the entropy change is a significant quantity that characterizes the human body from thermodynamic and holistic (i.e., considering a human body as a whole) viewpoints.

The human physiological entropy change could be considered as a composite measure of change in the whole physiological state in response to any external stimuli or stressor. However, if a single physiological indicator alone, such as the mean arterial pressure, can provide that information, then why do we need this entropy change as a composite measure of physiological response? The answer is as follows: the physiological concepts such as stimulus response (SR) specificity, organ response (OR) specificity, individual response specificity, and autonomic balance make the human physiological response a complex phenomenon [11]. The single physiological indicators, in this regard, provide a very narrow representation of the human physiological stress response system. It is only by recognizing the interaction among human subsystems in their response to any stressor stimuli that one could build a better model of human physiology. This study makes an effort to reduce the physiological complexity in terms of a composite entropy change. 


\section{Methodology}

3.1. Subjects. The data in the study were collected on eight subjects ( 5 male, 3 female). These subjects completed a standard psychophysiological stress profile procedure routinely used for assessment in the Human Engineering Laboratory at NASA Langley Research Center. The participants were all healthy (i.e., without any major health problems).

3.2. Data Collection. The physiological data were collected by a BioPac system via National Instrument LabView. The multiple physiological responses were collected from eight subjects who completed the 78-minute Physiological Stress Profile and included the two following conditions.

Condition 1 (relaxation period). Subjects relaxed in a semireclining position with eyes open for eighteen minutes listening to a guided relaxation tape. The physiological stress response data were collected. These eighteen-minute time-series data, $\mathrm{BP}_{\mathrm{O}}, \mathrm{HR}_{\mathrm{O}}, \mathrm{ST}_{\mathrm{O}}, \mathrm{EMG}_{\mathrm{O}}$, and $\mathrm{EDR}_{\mathrm{O}}$, were averaged and used in the physiological entropy change calculations as baseline data points.

Condition 2 (task period). Subjects completed a series of cognitive tasks presented by an oil refinery simulation program called Dexter. The primary goal of this simulation program was to examine the human physiological response to activities that induce boredom, drift in attention, and other negative effects on performance. The task lasted for sixty minutes and two data samples per minute were collected. Each one of these physiological measures $-\mathrm{BP}_{\text {Task }}, \mathrm{HR}_{\text {Task }}, \mathrm{ST}_{\text {Task}}$, $\mathrm{EMG}_{\text {Task}}$, and $\mathrm{EDR}_{\text {Task }}$ - was used in the calculations as shown in the next subsection.

3.3. Illustrative Example. Let us consider subject \#1; the eighteen-minute time-series data is averaged to get the following baseline values. Note that $\mathrm{BP}_{P, \mathrm{O}}$ and $V_{\mathrm{HR}, \mathrm{O}}$ are averaged and used in the physiological entropy generation calculations as baseline data points:

$$
\begin{aligned}
& \mathrm{BP}_{\mathrm{O}}(\mathrm{mmHg}): 118.74 . \\
& \mathrm{HR}_{\mathrm{O}}(\mathrm{bpm}): 68.49 . \\
& \mathrm{ST}_{O}\left({ }^{\circ} \mathrm{F}\right): 93.14 . \\
& \mathrm{EMG}_{O}(\mathrm{mV}): 334.17 . \\
& \operatorname{EDR}_{\mathrm{O}}(\mu \mathrm{mho}): 557.87 .
\end{aligned}
$$

Each one of the physiological measures, $\mathrm{BP}_{\text {Task }}, \mathrm{HR}_{\text {Task}}$, $\mathrm{ST}_{\text {Task }}, \mathrm{EMG}_{\text {Task }}$, and $\mathrm{EDR}_{\text {Task }}$, from the time-series is used to find the entropy change. Let us consider the values at the 5th minute during the task. A sample calculation of physiological entropy change at the 5 th minute for subject \#1 is shown as follows:

$$
\begin{aligned}
\Delta S_{\mathrm{BP}} & =\frac{\left(\mathrm{BP}_{\text {Task }}-\mathrm{BP}_{\mathrm{O}}\right) *\left(\mathrm{HR}_{\text {Task }}-\mathrm{HR}_{\mathrm{O}}\right)}{\left[\left(\mathrm{ST}_{\text {Task }}-\mathrm{ST}_{\mathrm{O}}\right) / 1.8\right]}, \\
\Delta S_{\mathrm{BP}} & =\frac{(136.05-118.74) *(81.97-68.49)}{\{(92.59-93.14) / 1.8-D\}} \\
& =-178.33 \mathrm{mmHg} \cdot \mathrm{bpm} / \mathrm{K} .
\end{aligned}
$$

The EMG-based entropy change is calculated as follows:

$$
\begin{aligned}
\Delta S_{\mathrm{EMG}} & =\frac{\left(\mathrm{EMG}_{\mathrm{Task}}-\mathrm{EMG}_{\mathrm{O}}\right) *\left(\mathrm{EDR}_{\text {Task }}-\mathrm{EDR}_{\mathrm{O}}\right)}{\left\{\left[\left(\mathrm{ST}_{\text {Task }}-\mathrm{ST}_{\mathrm{O}}\right) / 1.8\right] \pm D\right\}}, \\
\Delta S_{\mathrm{EMG}} & =\frac{(335.25-334.17) *(678.93-557.87)}{\{[(92.59-93.14) / 1.8] \pm D\}} \\
& =-100.21 \mathrm{mV} \cdot \mu \mathrm{mho} / \mathrm{K} .
\end{aligned}
$$

Note the following:

(1) Divide temperature difference $\Delta S T$ by 1.8 to get the units in Kelvin (K).

(2) The factor $D$ is used to deal with zeros and small temperature difference that would cause huge entropy change numbers. In this case, $D=-1$ since $\Delta \mathrm{ST}$ is negative. If it is positive then $D=+1$. This keeps the magnitude of the entropy change to within manageable limits while keeping the direction of temperature change congruent.

The above sample calculations are done for the entire task period of sixty minutes for each subject and repeated for all the eight subjects as illustrated in the next section.

\section{Results and Analysis}

The baseline data were obtained by averaging the eighteenminute relaxation period and are provided in Table 1 for the eight subjects. The $\Delta S_{\mathrm{BP}}$ and $\Delta S_{\mathrm{EMG}}$ results are presented in Figures 1 and 2.

Figures 1 and 2 quantitatively demonstrate key psychophysiological important concepts such as stimulus response (SR) specificity, organ response (OR) specificity, and individual (IR) response specificity [11]. For each subject, the response to the task varies within the sixty-minute period. For instance, in Figure 1, subject 4 shows normal variability with $\Delta S_{\mathrm{BP}}$ throughout the task. This is a demonstration of stimulus response (SR) specificity. In Figure 2, the same subject \#4 shows greater variability with $\Delta S_{\mathrm{EMG}}$ in the beginning of the task while learning to settle down as the task progresses. This is a demonstration of organ response (OR) specificity in which the subject is exhibiting higher reactivity via EMG and EDR combinations. Also, one can observe wide variations between individual subjects in both Figures 1 and 2, which demonstrates individual response (IR) specificity. Furthermore, the average entropy change value over a 60 minute time period was obtained for all eight subjects and shown graphically in Figure 3. It is observed that there is a wide variation among the subjects in terms of average entropy change response to the task demonstrating interindividual differences. These results demonstrate the importance of a thermodynamics-based approach to conducting any medical and/or clinical research involving human subjects.

It is clear from the results shown in Figure 3 that subjects show greater reactivity via entropy change $\left(\Delta S_{\mathrm{EMG}}\right)$ induced 

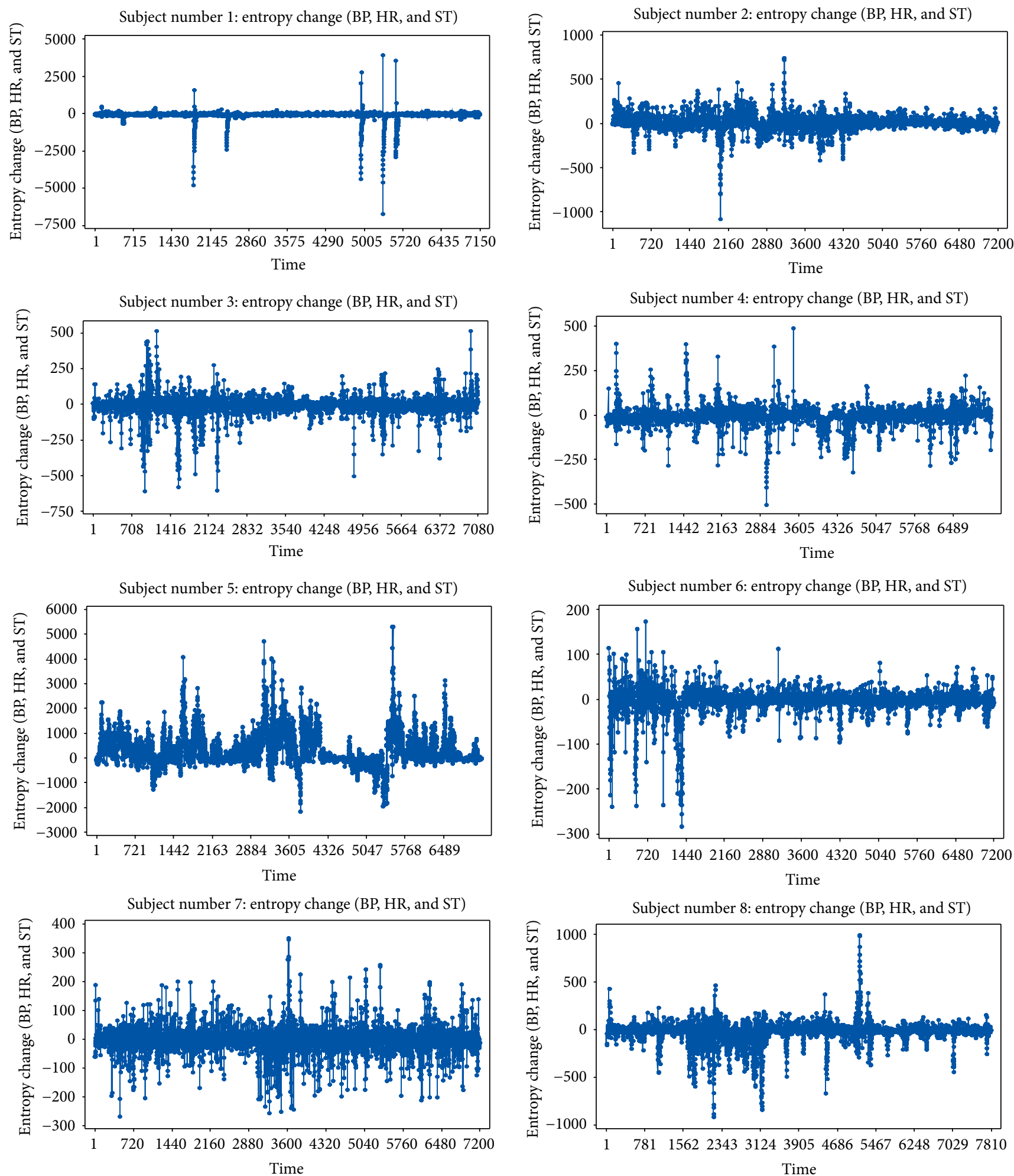

FIGURE 1: Entropy change $\left(\Delta S_{\mathrm{BP}}\right)$ across eight subjects: 1,6 , and 7 show significantly different patterns of variations compared to others.

by EMG and EDR than that by entropy change $\left(\Delta S_{\mathrm{BP}}\right)$ induced by blood pressure and heart rate. Both have temperature change as common denominators. Because of higher reactivity, $\Delta S_{\mathrm{EMG}}$ involving EMG and EDR measures need to be included in any comprehensive medical stress studies.

\section{Conclusion}

The study was based on the premise that Maxwell relations could be used to compute entropy change in terms of measurable physical variables. The experimental study involved 

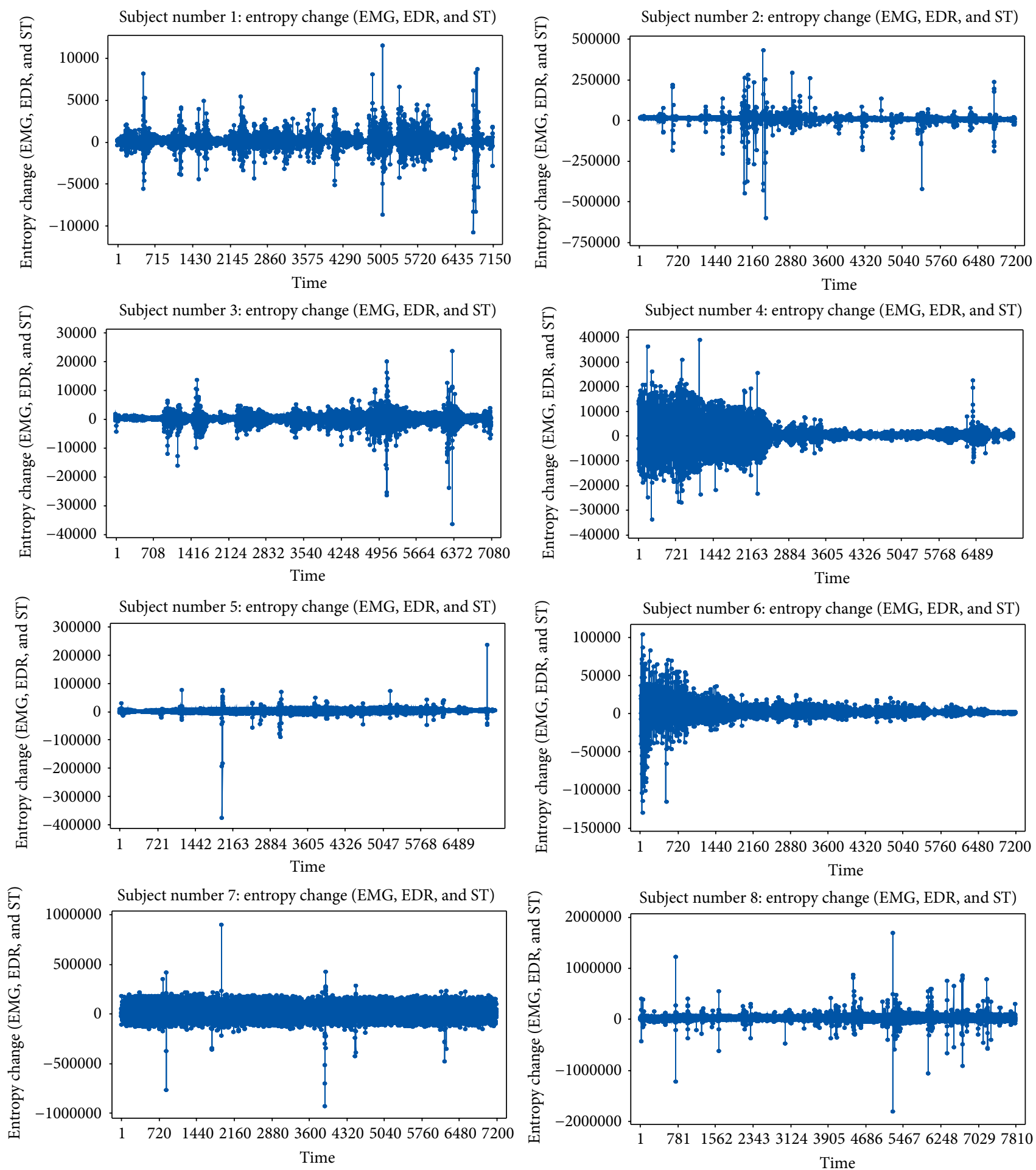

FIGURE 2: Entropy change $\left(\Delta S_{\text {EMG }}\right)$ across eight subjects: 2,5 , and 8 show significantly different patterns of variations while 7 exhibits a unique pattern.

eight subjects and was conducted in the Human Engineering Laboratory at NASA Langley Research Center. The physiological variables were measured at two states, relaxed and stressor task. The average physiological measures taken during relaxation were taken as the baseline values.
The physiological changes in blood pressure, heart rate, electromyogram, electrodermal response, and skin temperature from the reference (State 1) to stressor (State 2) were used in the Maxwell relations obtaining entropy changes, $\Delta S_{\mathrm{BP}}$, and $\Delta S_{\mathrm{EMG}}$, respectively. The results validate and verify key 
TABLE 1: Baseline values from the relaxation period for eight subjects.

\begin{tabular}{|c|c|c|c|c|c|}
\hline Subjects & $\mathrm{BP}_{\mathrm{O}}(\mathrm{mmHg})$ & $\mathrm{HR}_{\mathrm{O}}(\mathrm{bpm})$ & $\mathrm{ST}_{\mathrm{O}}\left({ }^{\circ} \mathrm{F}\right)$ & $\mathrm{EMG}_{\mathrm{O}}(\mathrm{mV})$ & $\mathrm{EDR}_{\mathrm{O}}(\mu \mathrm{mho})$ \\
\hline 1 & 118.74 & 68.49 & 93.14 & 334.17 & 557.87 \\
\hline 2 & 120.36 & 68.30 & 90.26 & 903.86 & 100.86 \\
\hline 3 & 144.37 & 44.64 & 83.72 & 418.81 & 52.69 \\
\hline 4 & 145.67 & 45.58 & 91.34 & 372.77 & 40.54 \\
\hline 5 & 135.90 & 67.85 & 90.92 & 897.79 & 20.00 \\
\hline 6 & 139.43 & 47.44 & 87.73 & 477.24 & 205.90 \\
\hline 7 & 140.17 & 73.16 & 75.64 & 373.94 & 381.62 \\
\hline 8 & 122.73 & 65.70 & 91.46 & 886.80 & 11.08 \\
\hline
\end{tabular}

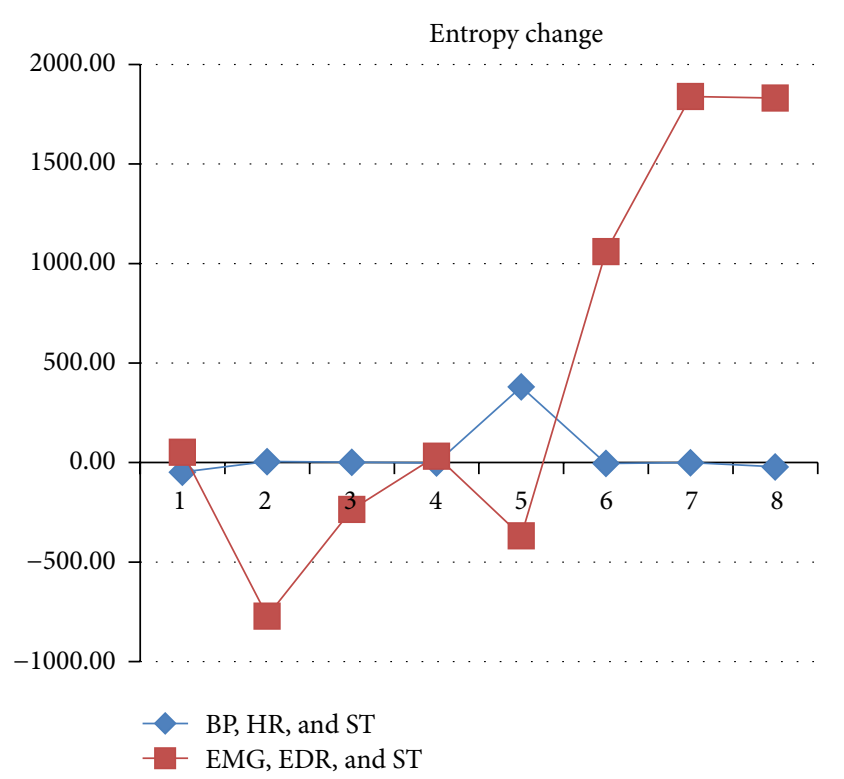

FIGURE 3: Average entropy change response in eight subjects.

psychophysiological concepts such as individual response (IR) specificity, organ response (OR) specificity, and stimulus response (IR) specificity.

The main purpose of this study was to use a novel thermodynamics-based approach to provide quantitative framework to assess human physiological stress responses. It is clear from the result that the modified Maxwell relations indeed provide something valuable to the biomedical engineering, medical, and health community, a quantitative measure of disorder or stress in human physiology. The entropy change could be postulated as disorder (probability) and incorporated in Shannon's entropy formulation [13] to link physical and informational aspects of human physiology. As this is beyond the scope of this paper, it could be valuable to the biomedical engineering community to utilize this concept for future applications. This fundamental concept could also lead to development of noninvasive, wearable sensors in order to gain insight into the prevention of human errors and occupational injuries and diseases. Future efforts will target field applications of this methodology. Specifically, the researchers plan to evaluate its usability and merit in occupational settings where workers are exposed to either physical (e.g., extreme temperatures, radiation), chemical
TABLE 2: Human biothermal-fluid system.

\begin{tabular}{lc}
\hline Physical variables & Biothermal-fluid system \\
\hline Pressure $(P)$ & Blood pressure $(\mathrm{BP})$ \\
Volume $(V)$ & Heart rate $(\mathrm{HR})$ \\
Temperature $(T)$ & Skin temperature $(\mathrm{ST})$ \\
Entropy $(S)$ & BP-based entropy $\left(S_{\mathrm{BP}}\right)$ \\
\hline
\end{tabular}

(e.g., particulate matter, organics solvents), or biological stressors (e.g., mold, fungi).

\section{Appendix}

\section{A. Using Maxwell Relations to Measure Entropy Change in Human Physiology}

A.1. Derivation of $\Delta S_{\mathrm{BP}}$ for Human Biothermal-Fluid System. The Maxwell relations were used to calculate entropy change $\left(\Delta S_{\mathrm{BP}}\right)$ in terms of measurable physical quantities such as pressure, volume, and temperature. An analogy between a simple physical piston-cylinder system and human physiological subsystem is conceptualized. The physical system characterized by pressure $(P)$, volume $(V)$, and temperature $(T)$ is mapped to that of a human biothermal-fluid system, blood pressure (BP), heart rate (HR), and skin temperature (ST), accordingly as shown in Table 2 .

A.1.1. Assumptions. (a) Human subphysiological system characterized by BP, HR, and ST is considered as a simple closed system from a macroscopic perspective for the purpose of this study. This makes it possible to apply Maxwell relations to examine human physiology.

(b) Blood pressure (BP), heart rate (HR), and skin temperature (ST) are equivalent to pressure $(P)$, volume $(V)$, and temperature $(T)$, respectively, as shown in Table 2.

A.1.2. Analysis. Let us consider a variable $z$ that is a continuous function of $x$ and $y$ :

$$
z=f(x, y) .
$$


It is convenient to write the above equation in the following form:

$$
d z=M d x+N d y
$$

where

$$
\begin{gathered}
M=\left(\frac{\partial z}{\partial x}\right)_{y} ; \\
N=\left(\frac{\partial z}{\partial y}\right)_{x} .
\end{gathered}
$$

If in (A.1), $x, y$, and $z$ are all point functions (i.e., quantities that depend only on the state and are independent of the path), the differentials are exact differentials. Therefore, in order for (A.1) to be an exact differential equation, the following condition must be satisfied:

$$
\left(\frac{\partial M}{\partial y}\right)_{y}=\left(\frac{\partial N}{\partial x}\right)_{y}
$$

Equation (A.4) is called the exactness condition.

Maxwell relations are derived from the property relations of thermodynamic potentials by invoking the exactness condition. For a simple blood pressure-based human physiological subsystem, there are four thermodynamic potentials:

$$
\begin{array}{r}
\text { Internal Energy: } d U_{\mathrm{BP}}=\mathrm{ST} d S_{\mathrm{BP}}-\mathrm{BP} d \mathrm{HR}, \\
\text { Enthalpy: } d H_{\mathrm{BP}}=\mathrm{ST} d S_{\mathrm{BP}}+\mathrm{HR} d \mathrm{BP},
\end{array}
$$

Helmholtz Function: $d F_{\mathrm{BP}}=-\mathrm{BP} d \mathrm{HR}-S_{\mathrm{BP}} d \mathrm{ST}, \quad(\mathrm{A} .5 \mathrm{c})$

$$
\text { Gibbs Function: } d G_{\mathrm{BP}}=\mathrm{HR} d \mathrm{BP}-S_{\mathrm{BP}} d \mathrm{ST} \text {. }
$$

The following Maxwell relations are obtained by invoking the exactness condition to the above four property relations:

$$
\begin{aligned}
& \left(\frac{\partial \mathrm{ST}}{\partial \mathrm{HR}}\right)_{\mathrm{S}_{\mathrm{BP}}}=-\left(\frac{\partial \mathrm{BP}}{\partial S_{\mathrm{BP}}}\right)_{\mathrm{HR}}, \\
& \left(\frac{\partial \mathrm{ST}}{\partial \mathrm{BP}}\right)_{\mathrm{S}_{\mathrm{BP}}}=\left(\frac{\partial \mathrm{HR}}{\partial S_{\mathrm{BP}}}\right)_{\mathrm{BP}}, \\
& \left(\frac{\partial \mathrm{BP}}{\partial \mathrm{ST}}\right)_{\mathrm{HR}}=\left(\frac{\partial S_{\mathrm{BP}}}{\partial \mathrm{HR}}\right)_{\mathrm{BP}}, \\
& \left(\frac{\partial \mathrm{HR}}{\partial \mathrm{ST}}\right)_{\mathrm{BP}}=-\left(\frac{\partial S_{\mathrm{BP}}}{\partial \mathrm{BP}}\right)_{\mathrm{ST}} .
\end{aligned}
$$

The above-mentioned partial derivatives are approximated to form a modified set of Maxwell relations that are used in
TABLE 3: Human biothermal-electric system.

\begin{tabular}{lc}
\hline Physical variables & Biothermal-electric system \\
\hline Tension $(\mathbb{T})$ & Electromyogram $(\mathrm{EMG})$ \\
Length $(L)$ & Electrodermal response $(\mathrm{EDR})$ \\
Temperature $(T)$ & Skin temperature $(\mathrm{ST})$ \\
Entropy $(S)$ & EMG-based entropy $\left(S_{\mathrm{EMG}}\right)$ \\
\hline
\end{tabular}

the present experimental study to compute the physiological entropy change:

$$
\begin{aligned}
& \left(\frac{\Delta \mathrm{ST}}{\Delta \mathrm{HR}}\right)_{\mathrm{S}_{\mathrm{BP}}}=-\left(\frac{\Delta \mathrm{BP}}{\Delta S_{\mathrm{BP}}}\right)_{\mathrm{HR}}, \\
& \left(\frac{\Delta \mathrm{ST}}{\Delta \mathrm{BP}}\right)_{S_{P}}=\left(\frac{\Delta \mathrm{HR}}{\Delta S_{\mathrm{BP}}}\right)_{\mathrm{BP}}, \\
& \left(\frac{\Delta \mathrm{BP}}{\Delta \mathrm{ST}}\right)_{\mathrm{HR}}=\left(\frac{\Delta S_{\mathrm{BP}}}{\Delta \mathrm{HR}}\right)_{\mathrm{BP}}, \\
& \left(\frac{\Delta \mathrm{HR}}{\Delta \mathrm{ST}}\right)_{\mathrm{BP}}=-\left(\frac{\Delta S_{\mathrm{BP}}}{\Delta \mathrm{BP}}\right)_{\mathrm{ST}} .
\end{aligned}
$$

Any of the above relations (A.7a)-(A.7d) could be used to quantify $\Delta S_{\mathrm{BP}}$, the human physiological entropy change. Using the Helmholtz function-based thermodynamic potential, the entropy change is given by

$$
\Delta S_{\mathrm{BP}}=\frac{\Delta \mathrm{BP} \times \Delta \mathrm{HR}}{\Delta \mathrm{ST}}
$$

A.2. Derivation of $\Delta S_{\mathrm{EMG}}$ for Human Biothermal-Electric System. The physical system characterized by tension $(\mathbb{})$, length $(L)$, and temperature $(T)$ is mapped to that of a human biothermal-electric system, electromyogram (EMG), electrodermal response (EDR), and skin temperature (ST), accordingly as shown in Table 3.

A.2.1. Assumptions. (a) Human subphysiological system characterized by EMG, EDR, and ST is considered as a simple closed system from a macroscopic perspective for the purpose of this study. This makes it possible to apply Maxwell relations to examine human physiology.

(b) Electromyogram (EMG), electrodermal response (EDR), and skin temperature (ST) are equivalent to tension $(\mathbb{\square})$, length $(L)$, and temperature $(\mathrm{ST})$, respectively.

A.2.2. Analysis. Maxwell relations are derived from the property relations of thermodynamic potentials by invoking the exactness condition. For a simple blood pressure-based 
human physiological subsystem, there are four thermodynamic potentials:

$$
\begin{aligned}
& \text { Internal Energy: } d U_{\mathrm{EMG}} \\
& \quad=\mathrm{ST} d S_{\mathrm{EMG}}-\mathrm{EMG} d \mathrm{EDR}, \\
& \text { Enthalpy: } d H_{\mathrm{EMG}}=\mathrm{ST} d S_{\mathrm{EMG}}+\mathrm{EDR} d \mathrm{EMG}, \\
& \text { Helmholtz Function: } d F_{\mathrm{EMG}} \\
& \quad=-\mathrm{EMG} d \mathrm{EDR}-S_{\mathrm{EMG}} d \mathrm{ST},
\end{aligned}
$$

Gibbs Function: $d G_{\mathrm{EMG}}$

$$
=\mathrm{EDR} d \mathrm{EMG}-S_{\mathrm{EMG}} d \mathrm{ST} \text {. }
$$

The following Maxwell relations are obtained by invoking the exactness condition to the above four property relations:

$$
\begin{aligned}
& \left(\frac{\partial \mathrm{ST}}{\partial \mathrm{EDR}}\right)_{S_{\mathrm{EMG}}}=-\left(\frac{\partial \mathrm{EMG}}{\partial S_{\mathrm{EMG}}}\right)_{\mathrm{EDR}}, \\
& \left(\frac{\partial \mathrm{ST}}{\partial \mathrm{EMG}}\right)_{\mathrm{S}_{\mathrm{EMG}}}=\left(\frac{\partial \mathrm{EDR}}{\partial S_{\mathrm{EMG}}}\right)_{\mathrm{EMG}}, \\
& \left(\frac{\partial \mathrm{EMG}}{\partial \mathrm{ST}}\right)_{\mathrm{EDR}}=\left(\frac{\partial S_{\mathrm{EMG}}}{\partial \mathrm{EDR}}\right)_{\mathrm{EMG}}, \\
& \left(\frac{\partial \mathrm{EDR}}{\partial \mathrm{ST}}\right)_{\mathrm{EMG}}=-\left(\frac{\partial S_{\mathrm{EMG}}}{\partial \mathrm{EMG}}\right)_{\mathrm{ST}} .
\end{aligned}
$$

The modified set of Maxwell relations that are used in the present experimental study to compute the physiological entropy change are

$$
\begin{aligned}
& \left(\frac{\Delta \mathrm{ST}}{\Delta \mathrm{EDR}}\right)_{S_{\mathrm{EMG}}}=-\left(\frac{\Delta \mathrm{EMG}}{\Delta S_{\mathrm{EMG}}}\right)_{\mathrm{EDR}}, \\
& \left(\frac{\Delta \mathrm{ST}}{\Delta \mathrm{EMG}}\right)_{S_{\mathrm{EMG}}}=\left(\frac{\Delta \mathrm{EDR}}{\Delta S_{\mathrm{EMG}}}\right)_{\mathrm{EMG}}, \\
& \left(\frac{\Delta \mathrm{EMG}}{\Delta \mathrm{ST}}\right)_{\mathrm{EDR}}=\left(\frac{\Delta S_{\mathrm{EMG}}}{\Delta \mathrm{EDR}}\right)_{\mathrm{EMG}}, \\
& \left(\frac{\Delta \mathrm{EDR}}{\Delta \mathrm{ST}}\right)_{\mathrm{EMG}}=-\left(\frac{\Delta S_{\mathrm{EMG}}}{\Delta \mathrm{EMG}}\right)_{\mathrm{ST}} .
\end{aligned}
$$

Using the Helmholtz function-based thermodynamic potential (A.11c), the entropy change is given by

$$
\Delta S_{\mathrm{EMG}}=\frac{\Delta \mathrm{EMG} \times \Delta \mathrm{EDR}}{\Delta \mathrm{ST}} .
$$

\section{Conflict of Interests}

The authors declare that there is no conflict of interests regarding the publication of this paper.

\section{Acknowledgment}

The authors wish to thank Dr. Alan Pope of NASA Langley Research Center for the initial support of this research project.

\section{References}

[1] P. W. Bridgman, The Nature of Thermodynamics, Harpers and Row, New York, NY, USA, 1961.

[2] I. Aoki, "Entropy flow and entropy production in the human body in basal conditions," Journal of Theoretical Biology, vol. 141, no. 1, pp. 11-21, 1989.

[3] I. Aoki, "Effects of exercise and chills on entropy production in human body," Journal of Theoretical Biology, vol. 145, no. 3, pp. 421-428, 1990.

[4] H. J. Morowitz, Entropy for Biologists, Academic Press, New York, NY, USA, 1970.

[5] H. J. Morowitz, Foundations of Bioenergetics, Academic Press, New York, NY, USA, 1978.

[6] L. Garby and P. S. Larsen, Bioenergetics: Its Thermodynamic Foundations, Cambridge University Press, Cambridge, UK, 1995.

[7] D. Jou and J. E. Llebot, Introduction to the Thermodynamics of Biological Processes, Prentice-Hall, Englewood Cliffs, NJ, USA, 1990.

[8] S. C. Boregowda, S. N. Tiwari, S. K. Chaturvedi, and D. R. Redondo, "Analysis and quantification of mental stress and fatigue using Maxwell relations from thermodynamics," Journal of Human Ergology, vol. 26, no. 1, pp. 7-16, 1997.

[9] S. C. Boregowda and W. Karwowski, "Modeling of human physiological stresses: a thermodynamics-based approach," Occupational Ergonomics, vol. 5, no. 4, pp. 235-248, 2005.

[10] H. Callen, Thermodynamics and an Introduction to Thermostatics, John Wiley \& Sons, New York, NY, USA, 2nd edition, 1985.

[11] J. L. Andreassi, Psychophysiology: Human Behavior and Physiological Stress Response, Lawrence Erlbaum Associates, Mahwah, NJ, USA, 4th edition, 2007.

[12] E. Schrodinger, What is Life? Cambridge University Press, Cambridge, UK, 1944.

[13] C. E. Shannon, "A mathematical theory of communication," The Bell System Technical Journal, vol. 27, no. 3, pp. 379-423, 1948. 

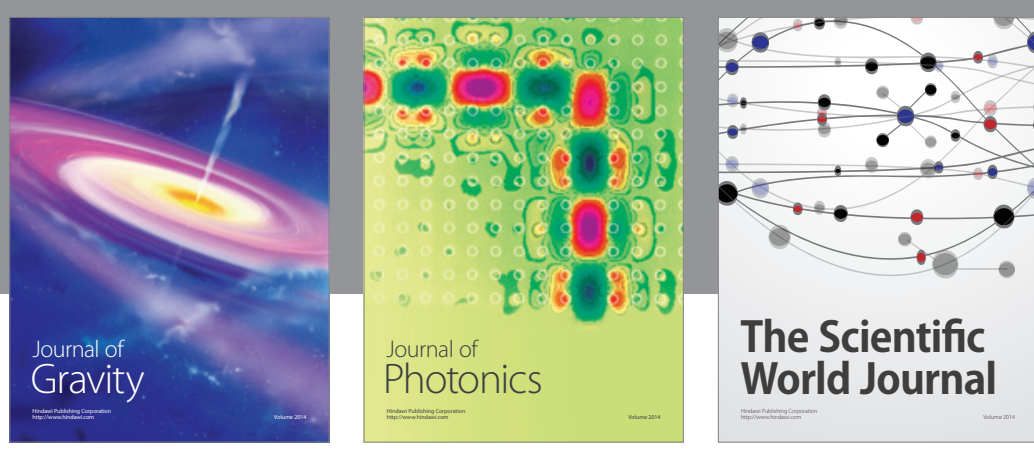

The Scientific World Journal
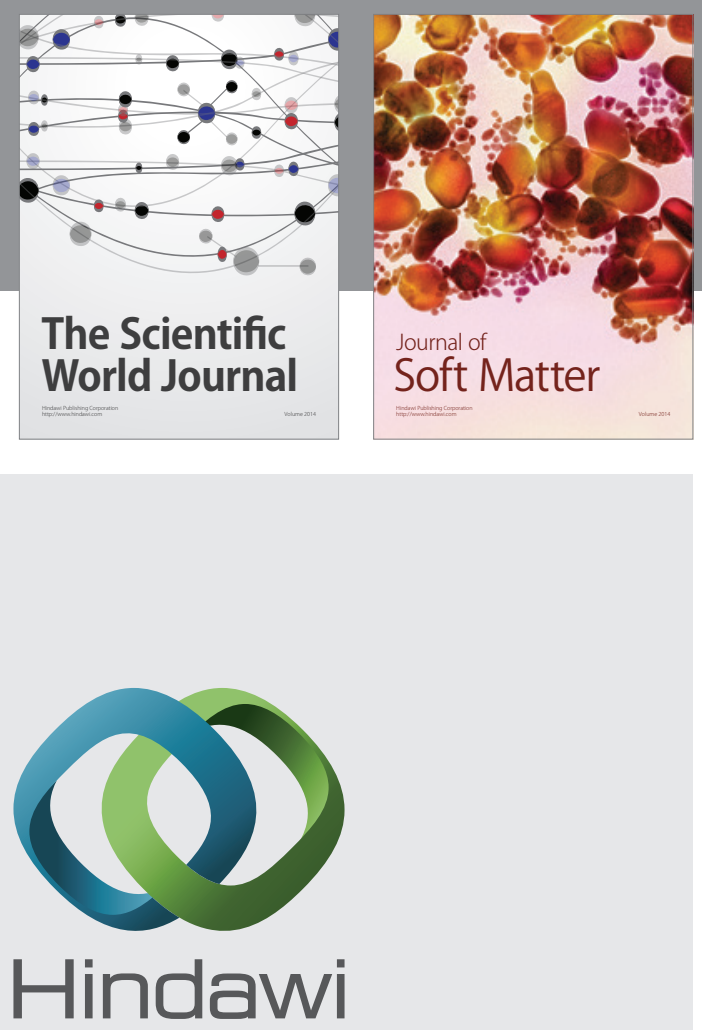

Submit your manuscripts at

http://www.hindawi.com

nternational Journal of

Statistical Mechanics
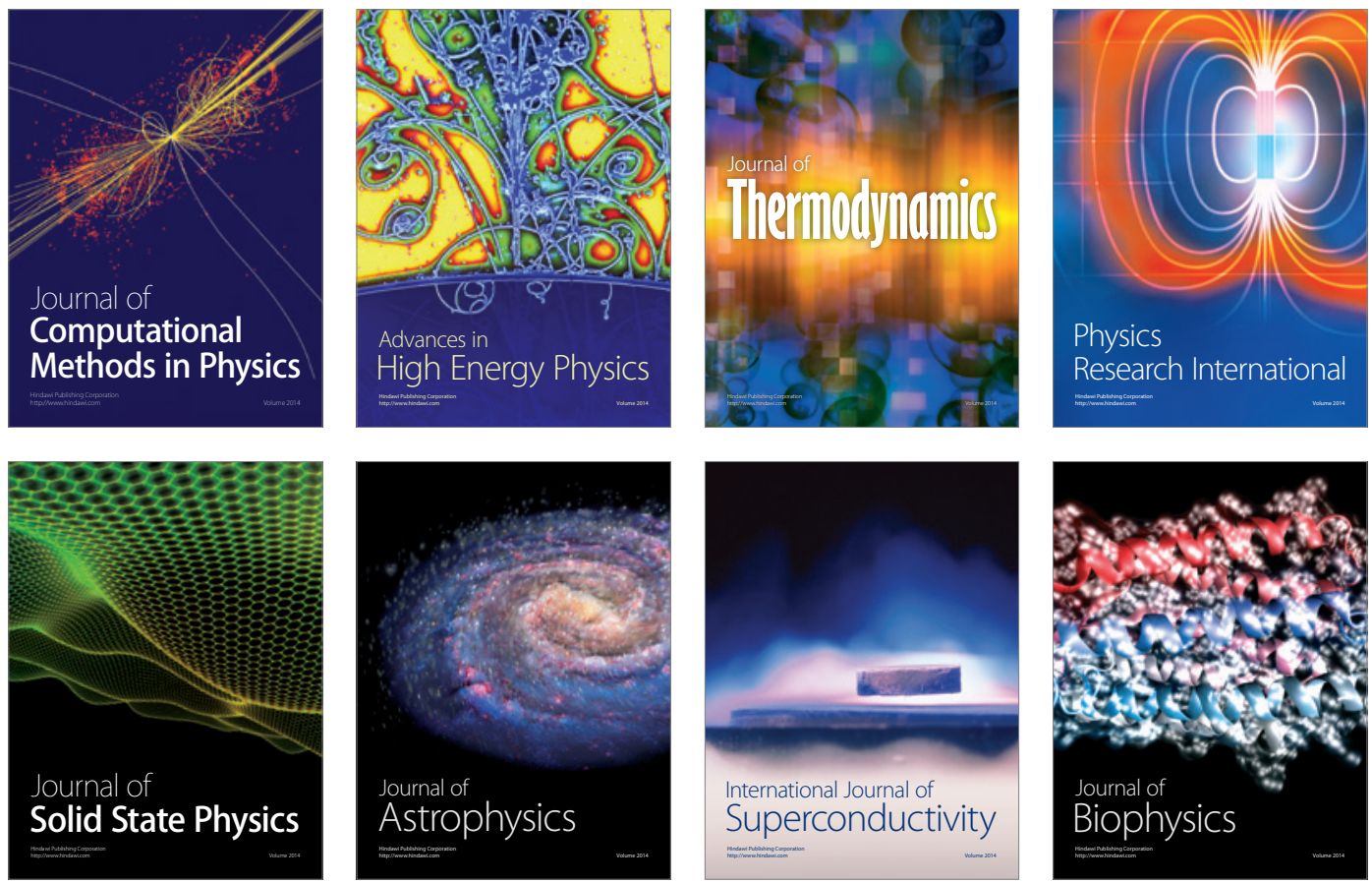
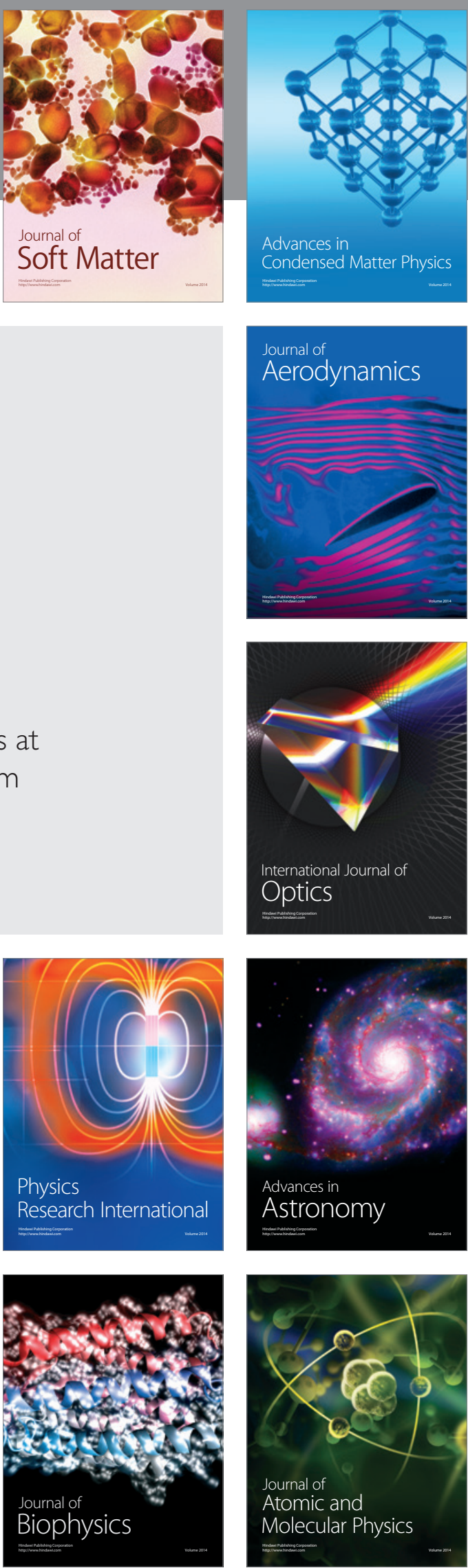